\title{
AMPLIANDO LA MIRADA. HACIA UN NUEVO ENFOQUE PARA LA REGULACIÓN DE OFERTA DE CARRERAS
}

\author{
Alberto Vásquez* \\ Gastón Zamorano**
}




\section{RESUMEN}

La regulación de la oferta de carreras en universidades en proceso de acreditación no debería ser distinta de la que opere para la educación superior en su conjunto. Tanto en los procesos de autorregulación que cada institución debe mantener, como en la regulación llevada a cabo por instancias externas, es necesario incorporar nuevos criterios y enfoques: a) preocuparse que el conjunto de la oferta de carreras mantenga niveles adecuados de calidad y pertinencia, no sólo de la creación de nuevos programas; b) profundizar los actuales enfoques de mercado, incorporando efectivamente la perspectiva de todos los usuarios del sistema y garantizar niveles mínimos de información pública sobre la oferta; c) complementar los criterios actuales, cambiando los ejes de análisis:desde carreras a proyectos académicos insertos en sus respectivos marcos institucionales, por un lado, y por otro, de la observación del "mercado de carreras" a una mirada desde la sociedad, para incorporar a todos los actores sociales y las temáticas relevantes para el país; $d$ ) asumir los nuevos desafíos para la educación superior que implican la globalización del sistema universitario, la incorporación de las tecnologías de la información y la transición hacia modalidades de formación continua.

\section{ABSTRACT}

Regulating the supply of degrees offered by Universities in the process of accreditation should be no different from the one for higher education as a whole. In the self-regulation process that each institution needs to have in place as well as in the regulation exercised by external agencies, new criteria and approaches need to be included: a) to see to it that the overall supply of degrees offered maintain an adequate level of quality and appropriateness, not only in terms of creating new programs; $b$ ) to further develop current market approaches by including the views of all the stakeholders in the system, ensuring minimum levels of public information on the available supply c) to supplement current criteria by changing the focus of analysis from university degrees to academic projects that are part of their respective institutional frameworks, on the one hand and, on the other hand, by changing the perspective of a "career market" to a society-based outlook so as to include all social actors and the major issues for the country; d) to address the new challenges faced by higher education which imply making the university system a global one, bringing in information technology systems and moving toward lifelong education methods. 


\section{AMPLIANDO LA MIRADA. HACIA UN NUEVO ENFOQUE PARA REGULAR LA OFERTA DE CARRERAS UNIVERSITARIAS}

En el modelo universitario que actualmente opera en el país, la oferta de carreras depende principalmente de las decisiones que se toman al interior de las instituciones de educación superior. Sólo las universidades que no cuentan con la autonomía que otorga el Consejo Superior de Educación, que representan una minoría, están sujetas a mecanismos de regulación externos y vinculantes. De este modo, para casi la totalidad del sistema, la regulación de la oferta de carreras descansa en los análisis internos de las propias universidades y en el control que la demanda y la competencia puedan ejercer.

Transcurridas dos décadas desde el ingreso de actores privados a la educación superior y a una década de la entrada en vigencia de la LOCE, existe consenso en que es necesario establecer instancias adicionales de regulación a la oferta de carreras. El desarrollo de mecanismos internos de regulación en las universidades ha sido lento y desigual. Actualmente, el mercado de educación superior presenta una serie de debilidades y asimetrías que desdibujan su capacidad de control sobre la oferta.

Hoy, cuando se comienzan a discutir y a realizar cambios en las políticas de educación superior en aspectos como el financiamiento, el sistema de ingreso, los sistemas de acreditación, los mejoramientos en la calidad y la equidad, no debe excluirse el tema de la oferta de carreras y su regulación. En este contexto, proponemos ampliar el enfoque con que este último tema se viene abordando, superando la mirada que se centra en las carreras como productos, que en forma agregada configurarían una oferta dentro de un mercado supuestamente homogéneo. Para ello, proponemos:

- Situar la discusión con miras a asegurar la calidad y la pertinencia;

- Perfeccionar el análisis del mercado;

- Incorporar criterios académicos; 
- Cambiar el eje desde el mercado a la sociedad;

- Mirar hacia el futuro;

Este enfoque debería aplicarse tanto en los procesos de autorregulación que cada institución debe generar, como en la regulación llevada a cabo por instancias externas. Al mismo tiempo, entendemos que la regulación de la oferta de carreras en universidades en proceso de acreditación, no debería ser distinta a la que opere para la educación superior en su conjunto. Toda discusión y propuesta al respecto debe pensarse y aplicarse con los mismos criterios para todas las instituciones de educación superior.

\section{ASEGURAMIENTO DE LA CALIDAD Y LA PERTINENCIA}

La preocupación por la regulación de la oferta se asocia al explosivo crecimiento de la matrícula que ofrecen las carreras de mayor demanda y a los peligros de saturación de profesionales que ello podría generar en el mercado laboral. A esto se suman las dudas sobre la calidad de la formación que algunas instituciones, sobre todo privadas y nuevas, estarían entregando a los alumnos en carreras de "tiza y pizarrón". De ahí que la regulación parece entenderse como un mecanismo para establecer restricciones a la creación de nuevos programas o al aumento de vacantes. Es decir, para limitar el crecimiento de la oferta.

Si bien la creación de nuevas carreras forma parte de los aspectos a incluir dentro del diseño de mecanismos de regulación, el horizonte debe ser el aseguramiento de la calidad de la oferta de todo el sistema. Esto implica, por un lado, incluir en la regulación a todos los programas ofrecidos y a todas las instituciones, sin distinción. Por otro, significa concebir la evaluación como un proceso de seguimiento permanente de la calidad de las carreras.

Si la calidad es el criterio principal para regular la oferta al interior del sistema, el segundo aspecto a considerar es la pertinencia social, entendida ésta como la adecuación a las necesidades del entorno regional, nacional e internacional. Nuevamente, este factor no debe considerarse sólo al momento de aprobar una carrera nueva, sino que debe traducirse en la revisión permanente de los programas que se dictan: sus contenidos, metodologías y perfiles y empleabilidad de los egresados. 


\section{EL ANÁLISIS DE MERCADO: IMPORTANCIA DE LA INFORMACIÓN Y DE LOS USUARIOS}

El diagnóstico de los problemas y necesidades de regulación de la oferta de carreras se ha hecho desde la perspectiva y el lenguaje del "mercado", en el cual suele prevalecer un análisis simplificador que no distingue las variaciones y complejidades que actualmente existen.

En primer lugar hay que poner atención a la amplia diversidad de la oferta y la demanda, junto al explosivo crecimiento del mercado de la educación superior en estos últimos 20 años. La imagen de homogeneidad y estabilidad que presentaba hace dos décadas ya no sirve de referencia para pensar los mecanismos de regulación actuales. Estamos ante un mercado altamente segmentado, donde las oportunidades de elección se han ampliado en forma diferenciada. Por ejemplo, la oferta de carreras para un joven recién egresado de la enseñanza media no es la misma si tiene 700, 600 o 500 puntos en la PAA. Tampoco es la misma para quienes residen en regiones o bien en Santiago (e incluso en distintas comunas de la capital), ni para quienes provienen de familias con distinta condición socioeconómica. Se han creado nuevos nichos: carreras vespertinas, programas a distancia, programas para trabajadores, carreras de segunda titulación en dos años, etcétera. En este contexto la competencia por la matrícula entre instituciones también es fragmentada y focalizada: no todas las universidades compiten entre sí y aquellas que lo hacen, no actúan de esa forma en todos los programas. El creciente despliegue publicitario a que nos estamos acostumbrando, forma parte y refleja la actual diversidad y asimetría de la competencia.

Este panorama obliga a hacerse cargo de la diversidad y a dedicar mayores esfuerzos al estudio de las características y tendencias de este complejo mercado, para poder diseñar mecanismos de regulación efectivos.

Por otro lado, al centrar las preocupaciones en el lado de la oferta se ha dejado en segundo plano la perspectiva de la demanda. El diseño de mecanismos de regulación debe incorporar de modo adecuado la perspectiva de todos los usuarios del sistema: postulantes y sus familias, estudiantes, académicos, instituciones, egresados y titulados, 
empleadores, incluso de los encargados de la planificación gubernamental. Tanto dentro de las universidades como entre los observadores y reguladores centrales, son escasas las mediciones y los estudios de las expectativas, demandas o grados de satisfacción de los distintos actores respecto de la oferta educativa. En las ocasiones en que se realizan, persisten los enfoques reduccionistas, ya sea porque se centran en un solo tipo de usuario, o bien porque no reconocen segmentación alguna dentro de la demanda.

Un tema sensible en este sentido radica en la información sobre la oferta. La creciente complejidad del sistema no ha ido acompañada de la generación y difusión de información relevante para la toma de decisiones de los usuarios. Este es uno de los aspectos que menos desarrollo ha tenido en el mercado universitario. Hoy, todo parece depender del capital que cada universidad posea para realizar grandes inversiones en publicidad, lo que limita y distorsiona seriamente el control que debería ejercer la demanda sobre la oferta. Garantizar y entregar niveles mínimos de información pública sobre las carreras y promover su uso, debiera ser una de las tareas centrales de las instancias gubernamentales que se ocupen de la regulación.

Ciertamente la falta de información pública seria, transparente, válida y fidedigna es actualmente un claro déficit en el sistema de educación superior. Estamos ante un mercado dinámico y altamente complejo, pero al mismo tiempo, escasamente estudiado, desigualmente desarrollado y distorsionado por los impactos publicitarios y el poder económico con que se cuenta para financiarlo.

\section{INCORPORAR CRITERIOS ACADÉMICOS}

La incorporación de criterios académicos es vital para el desarrollo de un buen sistema de regulación. Mirado desde el interior de las universidades, este objetivo se dificulta cuando la atención se centra exclusivamente en las carreras consideradas como productos que cada institución coloca en el mercado de la educación superior, aislándolas del entorno institucional en que se desarrolla cada programa. Cuando se incluyen variables institucionales en la construcción de indicadores de calidad para las carreras (como en I.N.D.I.C.E.S. ), éstos se limitan 
a factores de infraestructura y recursos. Pocas veces se revisan aspectos como la existencia de una masa crítica de académicos, su relación con las líneas de investigación departamental e institucional, con los procesos de planificación de la producción académica en su conjunto, o su coherencia con la misión de la universidad que la ofrece.

Desde el ángulo académico, el foco de la evaluación, sobre todo en el diseño de mecanismos de autorregulación dentro de las universidades, debe trasladarse de las "carreras" a "proyectos académicos", insertos en sus respectivos marcos y procesos institucionales. La tarea de una instancia de regulación externa de la oferta de carreras debe ser, por un lado, incorporar indicadores que den cuenta de la consistencia de estos proyectos académicos, principalmente de tipo cualitativo. Por otro lado, promover y asesorar a las instituciones de educación superior para que diseñen e implanten sistemas de autoevaluación claramente académicos, puesto que en última instancia son las propias universidades las que deben regular con seriedad su oferta.

La planificación académica al interior de una universidad, en su vertiente de oferta educativa, debe comenzar por interrogar a las demandas del desarrollo, la evolución de los conocimientos, las transformaciones de los oficios profesionales, las posibilidades de empleo, las necesidades sociales y los quiebres culturales que desea atender mediante las carreras que se ofrecen. En fidelidad a su misión institucional, la oferta necesita examinarse en función de las fortalezas que posea y de la masa crítica de conocimientos y experiencia de sus equipos académicos. Pero también, exigidos por un mejor servicio, hay que examinar la oferta en función de las potencialidades que aparecen al explorar nuevas modalidades de formación, que superen los estilos clásicos de transmisión de conocimientos. Asimismo, implica como actitud básica evitar la apertura de carreras guiados solamente por criterios económicos.

La planificación de la oferta educativa debe ser congruente con la misión institucional y con las definiciones que se acuerden sobre el modelo de universidad que se desea concretar. En este sentido, una universidad no puede concebirse como la simple suma de carreras y 
posgrados, ni puede medirse por el número de alumnos, o los metros cuadrados de sus instalaciones. Un proyecto universitario será siempre un asunto complejo, cualitativo y dinámico. Las universidades puramente docentes son verdaderas fábricas de licenciados y se transforman en parte del problema y no de la solución de nuestra sociedad.

Por otro lado, la universidad debe rescatar y mantener su papel educativo y formador de personas. Resulta decisivo prestar atención al tipo de formación que entrega y a las modalidades pedagógicas que usa en los procesos de enseñanza y aprendizaje. Se requiere de modalidades dedicadas al desarrollo de competencias y valores en los jóvenes universitarios: no se trata solamente de "formar profesionales", sino de formar hombres y mujeres competentes, comprometidos con su sociedad, con sentido de servicio. En consecuencia, el modelo pedagógico que se utilice debería ser parte de los criterios a considerar para la regulación y evaluación de las carreras.

Planificar nuevas carreras implica, también, considerar los diagnósticos actualizados acerca de la demanda y la oferta de educación superior en el país y en la ciudad en que se trabaja. Asimismo, implica hacerse cargo de los estados del conocimiento y de su constante y rápida evolución hacia áreas de conocimiento cada vez más interrelacionadas y estructuradas en función de objetos de conocimiento, dirigidas a responder frente a determinados segmentos de la realidad. En consecuencia, la racionalidad de planificar en función de carreras acotadas en campos disciplinarios parece ser parte del pasado. No responde al estado actual de las operaciones que hoy es preciso realizar con el conocimiento, en el ámbito de la educación superior (generarlo, depurarlo, conservarlo, transmitirlo, aplicarlo).

Es preciso concebir la oferta educativa como un movimiento de consolidación, transformación y desarrollo académico en torno a áreas de conocimiento, examinadas y aprobadas en pertinencia al proyecto universitario. Se trata, en suma, de promover una cultura académica iluminada por los valores institucionales y por el análisis permanente de la realidad y sus demandas, para ofrecer respuestas válidas y posibles a los desafíos que nos presenta la sociedad nacional. 


\section{UNA MIRADA DESDE LA SOCIEDAD Y LOS NUEVOS DESAFÍOS}

Si al interior de las instituciones se debe cambiar el foco de análisis desde carreras aisladas a proyectos académicos insertos en su entorno institucional, en la observación del sistema de educación superior la mirada debe ampliarse desde el mercado a la sociedad.

Los criterios y definiciones de un sistema de regulación de la oferta de carreras tienen que estar insertos y ser compatibles con las políticas de educación superior. De igual modo, se debe dejar espacio para que actores ajenos al ámbito universitario puedan incorporar sus perspectivas, a fin de dar cabida a las distintas necesidades e intereses del país. Todo esto no es posible si la regulación se aplica solamente al mercado y sus variables. De esa forma se obtienen solamente mecanismos o sistemas de regulación, pero no lo que necesitamos: una política de regulación con visión de conjunto.

En esta perspectiva es posible plantearse aspectos como el rol del Estado, la autonomía institucional y académica de las universidades, las necesidades locales y regionales, y la vinculación de la regulación con el financiamiento de la educación superior. Ningún sistema de regulación es neutro, ni en sus supuestos ni en sus consecuencias, de modo que siempre debe poseer el respaldo de una discusión sustantiva respecto de los grandes objetivos e implicaciones que tiene su aplicación.

Por ejemplo, es un objetivo estratégico para el desarrollo del país contar con profesores de enseñanza básica y media en calidad y cantidad adecuadas a los cambios que se están implementando y a los desafíos que enfrenta la educación. Para atender este objetivo, no basta con la lógica del mercado: en general los jóvenes de altos puntajes no postulan a carreras pedagógicas. Se requiere, por lo tanto, del concurso de las instituciones de educación superior, de políticas públicas con claros incentivos, del apoyo de las autoridades nacionales, regionales y locales. Solamente mediante el compromiso de diversos actores sociales se podrá responder adecuadamente a dicho objetivo. Este caso ilustra la afirmación de que la regulación de la oferta de carreras debe concebirse en el marco de una política general y no puede sólo quedar sometida a la regulación del mercado. 
Por último, una política de regulación de la oferta de carreras debe comenzar a pensar en los nuevos desafíos que enfrenta la educación superior. Por ejemplo, la progresiva globalización del mercado del sistema universitario implica dar respuestas a fenómenos como la oferta de programas dictados por universidades internacionales. De igual modo, la oferta de programas a distancia vía internet, el surgimiento de programas de corta duración dirigidos a regularizar estudios anteriores o a obtener una segunda titulación, deben dar paso al diseño de nuevas estrategias de regulación externa e interna. El conjunto del sistema universitario se encuentra en un proceso de transición hacia modalidades que están aún esbozándose, como la formación continua y la incorporación de las tecnologías de la información que rompe barreras institucionales y nacionales. La regulación debe ser lo suficientemente flexible como para, al menos, no impedir que las universidades nacionales puedan desarrollar sus propias estrategias destinadas a enfrentar estos cambios.

Los avances realizados por la Comisión Nacional de Acreditación de Programas de Pregrado son positivos. La regulación de la oferta de carreras debe insertarse en este proceso, ojalá dentro de una modalidad de certificación que incorpore los criterios de política nacional y que sea compatible con estándares internacionales. El sistema de acreditación debe ser flexible y no centralizado, promoviendo la convivencia (y equivalencia) de varios sistemas: internos, externos, públicos, privados, nacionales e internacionales.

Al mismo tiempo, la construcción de una agenda de investigación que permita entregar mayor claridad para construir una política de regulación acorde con la realidad del sistema y el mercado universitario, constituye una tarea urgente. 


\section{FORMACIÓN CONTINUA Y A DISTANCIA: UNA VISIÓN A PARTIR DE LA EXPERIENCIA}

Fidel Oteiza*

Juan Silva**

* Director del Centro Comenius, Universidad de Santiago de Chile.

** Encargado del área pedagógica del Centro Comenius, Universidad de Santiago de Chile. 